\title{
Transport maritime conteneurisé et mondialisation
}

\author{
Antoine Frémont \\ Martin Soppé \\ Université du Havre \\ FRE 2795 I.D.E.E.S/CIRTAI \\ Action Concertée Incitative "Etude comparée des flux conteneurisés en \\ Europe et en Asie orientale"
}

In Annales de géographie, 2005 , n642, pp. 187-200. 


\section{Introduction}

Objet de tant de débats contradictoires, la mondialisation s’invite chaque jour chez nous, qu'on le veuille ou non : un café sud-américain, une paire de chaussures de sport ou un jouet conçus aux Etats-Unis et fabriqués en Chine sont consommés à bas prix dans chaque ville d'Europe sans que les déplacements de plusieurs milliers de kilomètres effectués par ces marchandises, avec leurs problèmes de coûts, de temps, de pollutions ou autres aléas éventuels, ne suscitent la moindre interrogation chez les clients finaux ou intermédiaires. Ces déplacements sont pleinement intégrés dans les processus de production, de vente ou de consommation à travers la logistique. Pourtant, à l'inverse des flux financiers ou d'informations, les flux du commerce international ne sont pas immatériels mais reposent sur une organisation lourde qui se déploie à l'échelle mondiale : le transport maritime. La croissance des échanges de produits manufacturés à travers le monde n’a été rendue possible que par la conteneurisation, branche spécifique du transport maritime, et qui, par son efficacité, constitue l'épine dorsale logistique de la mondialisation.

Il existe une interdépendance croissante entre la conteneurisation, qui est au service du commerce international, et l'économie mondiale du fait du processus de globalisation. Mais le transport maritime conteneurisé dispose aussi de ses propres règles de fonctionnement et d'organisation, indépendantes de celles du commerce international. Sa puissance est étudiée à travers l'analyse systématique de l'offre de transport des plus grands armements conteneurisés mondiaux, ce qui permet de dresser un schéma général de la circulation maritime du trafic conteneurisé et de définir les principales caractéristiques spatiales de cette activité.

\section{Une activité vitale, un secteur mal connu}

\subsection{Laconteneurisation : épine dorsale de la mondialisation}

La croissance presque parallèle, depuis la fin de la Seconde Guerre mondiale, du commerce mondial en volume, des tonnages transportés par la voie maritime et de la flotte mondiale, illustre bien les relations d'interdépendance qui existent entre le transport maritime et l'économie (figure 1). Alors que les exportations mondiales ont enregistré une croissance en volume de l'ordre de 6,2\% par an, les échanges par voie 
maritime sont passés de 550 millions de tonnes en 1950 à 5,5 milliards de tonnes en 2002, soit une croissance moyenne annuelle de 4,5\% par an. Pendant la même période, la flotte mondiale a quintuplé, de $84000 \mathrm{TJB}^{\mathbf{1}}$ à 537000 TJB, soit une croissance moyenne annuelle de 3,6\%.

Figure 1. Indices des exportations mondiales (volume), du transport maritime (tonnes), de la flotte mondiale (TJB) et de la flotte des portes-conteneurs intégraux (TJB) entre 1950 et 1999

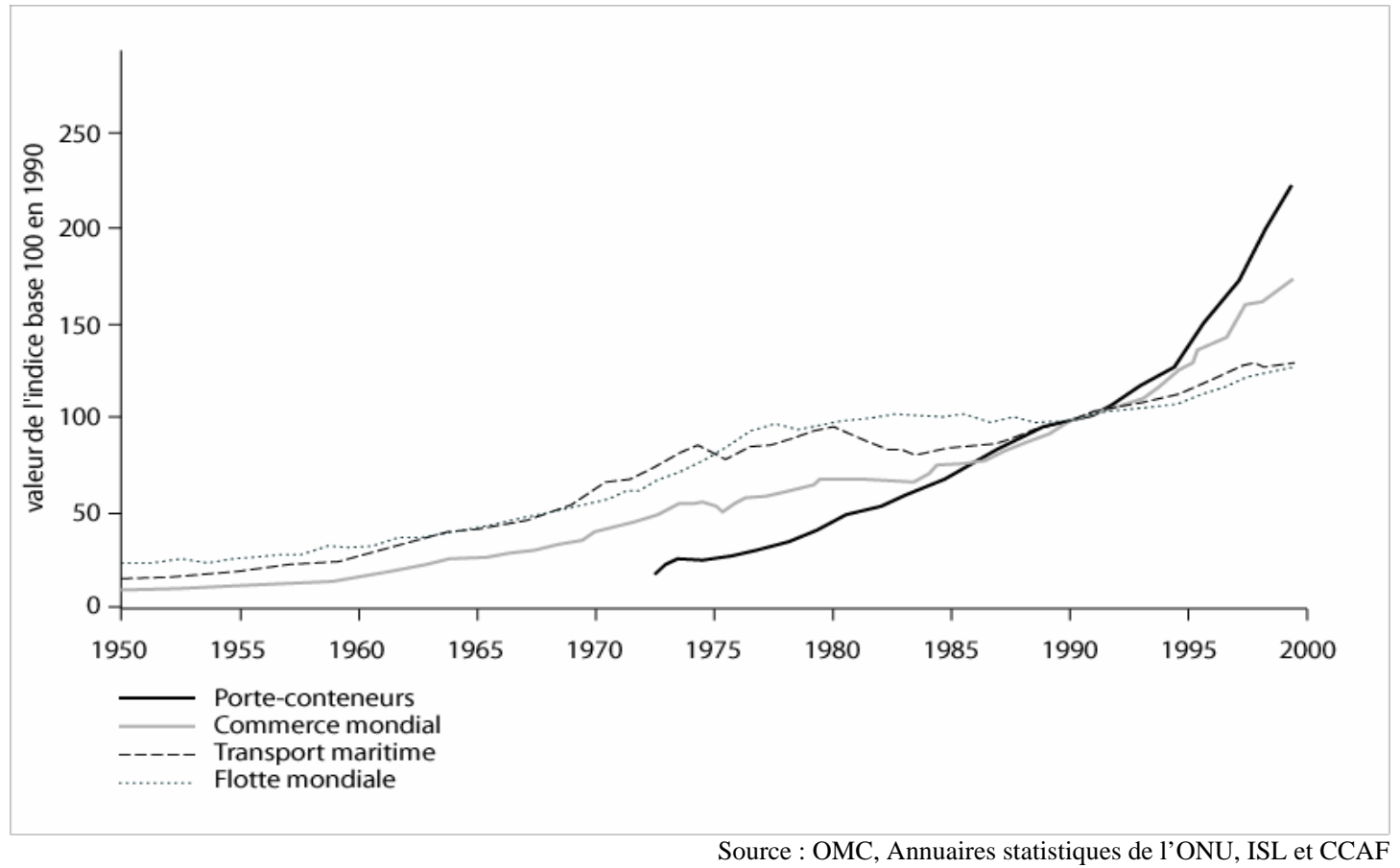

De la fin de la Seconde Guerre mondiale aux deux chocs pétroliers de 1973 et 1979, la croissance économique des pays développés, fondée sur les industries lourdes et leur capacité à importer massivement des matières premières, se traduit par une part prépondérante du pétrole dans le total des marchandises transportées par la voie maritime (54\% en 1979) et des pétroliers au sein de la flotte mondiale (42\%) (figure2).

\footnotetext{
${ }^{1}$ Le tonneau de jauge brute (TJB) est une unité de mesure utilisée pour exprimer la capacité d'un navire en volume. Un tonneau est égal à 2,83 $\mathrm{m}^{3}$.
} 
Figure 2. Transport par voie maritime des grands types de marchandises, en milliards de tonnes, 1960-2000

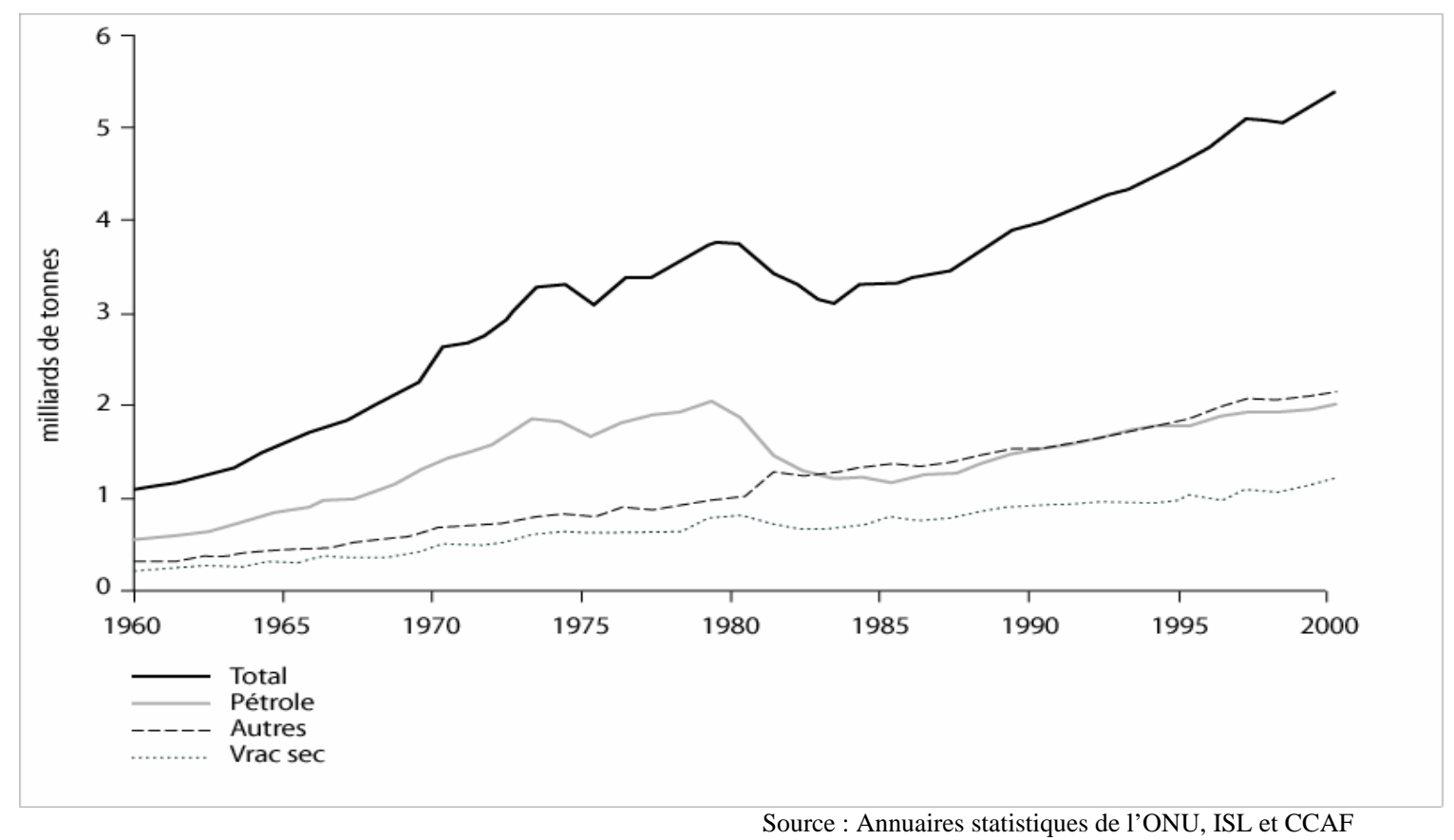

A partir des années 1970, les échanges de produits manufacturés deviennent le moteur principal de la croissance du commerce international. Leur part représente aujourd'hui, en valeur, environ $76 \%$ du total des exportations mondiales contre 62\% en 1970. Ces échanges constituent l'essentiel de l'activité d'exportation des pays riches de l'OCDE : 80\% en valeur aujourd'hui contre environ 70\% en 1970.

Cette croissance en valeur se répercute en volume dans la croissance des échanges par voie maritime des autres marchandises qui comprennent l'ensemble des marchandises qui ne sont pas en vrac. Alors que le trafic des vracs n'augmente que de $1,7 \%$ en moyenne par an depuis 1970, cette croissance est de 3,9\% pour les autres marchandises. Avec 2,3 milliards de tonnes d'autres marchandises transportées par la voie maritime en 2002 contre seulement 671 en 1970, leur part dans le trafic total est de $41 \%$ contre 26\% en 1970 (figure 2).

La conteneurisation a rendu techniquement possible cette formidable expansion. Appliquée au transport océanique, notamment sur l’Atlantique 
Nord à partir de 1965, étendue ensuite à l'échelle de la planète, elle permet l'acheminement des marchandises diverses dans des contenants aux dimensions standardisées ${ }^{3}$. Elle se traduit par de formidables gains de productivité. La capacité de transport des porte-conteneurs ne cesse d'augmenter : elle est aujourd'hui de plus de 8000 EVP pour les plus gros d'entre eux alors qu'elle ne dépassait pas 1000 EVP au début des années 1970. Un porte-conteneur de $6000 \mathrm{EVP}$, avec un équipage d'une vingtaine d’hommes au plus, a une capacité de transport environ vingt fois supérieure à celle d'un cargo conventionnel des années cinquante ou soixante dont l'équipage s’élevait à une quarantaine de personnes. La manutention de ces navires ne dure que quelques heures car plusieurs portiques à conteneurs, manipulant chacun de 20 à 25 conteneurs à l'heure, peuvent être affectés simultanément aux opérations de chargement/déchargement d'un même navire. Les coûts de transport sont aujourd'hui si faibles que la conteneurisation s'étend à des produits jusque là transportés en vrac comme le sucre ou les céréales.

Le transport conteneurisé s’insère dans les réseaux de production et de distribution à une échelle mondiale. Outil intermodal, le conteneur permet une livraison des marchandises porte à porte, de l'entreprise au magasin de distribution, en ajustant avec précision les quantités: le transport intègre la chaîne logistique qui participe au processus de création de la richesse produite. Les organisateurs du transport, notamment les armateurs de lignes régulières qui exploitent les portes-conteneurs et gèrent les parcs de conteneurs, développent des logiques propres d’organisation afin d'optimiser le fonctionnement des réseaux de transport maritimes et terrestres.

La conteneurisation a atteint aujourd'hui des dimensions considérables. Elle concentre $80 \%$ du trafic des marchandises diverses par la voie maritime, demain plus encore. Les portes-conteneurs intégraux représentent $11 \%$ de la flotte mondiale (mesurée en TJB) en 2001, contre seulement $6 \%$ en 1990 et $3 \%$ en 1980 . La capacité de transport est de près

\footnotetext{
${ }^{3}$ On distingue deux principaux types de conteneurs. Le conteneur de 20 pieds est long de $6,058 \mathrm{~m}$, large de 2,438 m et haut de 2,6 m. Sa capacité est de l'ordre de 21,5 tonnes. Le conteneur de 40 pieds a une longueur double de celle du conteneur de 20 pieds. On mesure
} 
de 7 millions d'EVP contre 771000 en 1980, soit une croissance moyenne annuelle de 11\% ! Enfin, on peut estimer à plus de 230 millions le nombre d’EVP manutentionnés dans les ports du monde en 2001, contre 83 millions en 1990, 35 millions en 1980 et un peu plus de 4 millions en $1970 \ldots$ Si le transport maritime conteneurisé reste un service qui permet les échanges internationaux, son organisation relève désormais d'une logique industrielle afin d'assurer des dessertes maritimes efficaces, qui acquièrent leur propre cohérence en dehors même du système des échanges internationaux et nécessitent une très forte intensité capitalistique.

\subsection{Une étude de l'offre de transport conteneurisé}

Les données statistiques pour apprécier les flux de conteneurs à travers le monde sont pratiquement inexistantes. L'administration américaine en propose mais pour les seuls ports des Etats-Unis. La seule ressource qui permet des comparaisons à l'échelle internationale se limite aux données portuaires, principalement le nombre de conteneurs manutentionnés dans chaque port. Ces chiffres donnent une information sur le poids de chaque port mais ne disent rien sur les flux de conteneurs entre les ports, ni sur l'implication de tel ou tel armement sur une route maritime et sa part dans les trafics.

Pour remédier à cette lacune et à défaut de disposer de données sur les quantités transportées par les armements, informations qui sont confidentielles, nous avons analysé, pour l’année 2002, l’offre commerciale de transport des 26 premiers armements mondiaux à partir de la publication annuelle Containerisation international Yearbook 2003 qui fait référence dans la profession. Les informations fournies ont été dans la mesure du possible recoupées avec celles publiées sur les sites web des différents armements pris en compte. Ceux-ci concentrent 61,5\% de la capacité mondiale de la flotte des portes-conteneurs (tableau 1). En additionnant les capacités en EVP, pour les ports ou pour les routes maritimes, nous obtenons la Capacité Hebdomadaire de Transport Conteneurisé (CHTC) offerte par les armements dans ces ports ou sur ces routes. Le passage d'un navire de 4000 EVP dans un port ne signifie pas que les 4000 EVP du

les capacités de transport des navires ou le nombre de «boîtes » manutentionnées dans les ports en EVP. 1 EVP vaut un conteneur de 20 pieds. 1 conteneur de 40 pieds vaut 2 EVP. 
navire sont manutentionnés. La manutention ne porte que sur une partie de cette capacité. Il s'agit donc bien d'une capacité théorique offerte, plus ou moins utilisée en fonction des ports.

Les fréquences de service offertes par les armements étant généralement hebdomadaires, toutes les capacités ont été ramenées, par souci de cohérence, à une base commune d'une semaine. Ces différentes données ont été réunies au sein d'une base de données sur la Capacité Hebdomadaire de Transport Conteneurisé (base CHTC). L'exploitation de la base CHTC permet une triple approche : par armement, par port et par relation entre les ports. En fonction de la problématique choisie, des regroupements peuvent être effectués au sein de ces trois catégories.

\section{Un système global et hiérarchisé}

\subsection{L'Asie orientale au cour du système}

L’Asie orientale est aujourd'hui au cœur du système de transport maritime conteneurisé dans le monde. Sur les 26 premiers armements mondiaux étudiés, 16 appartiennent à cette région du monde. Ils totalisent $53,7 \%$ de la capacité de transport de ces 26 premiers armements et participent pour plus de $30 \%$ à la capacité de la flotte conteneurisée mondiale. Leur croissance s’est effectuée au cours des années 1980. En 1979, seuls quatre armements étaient asiatiques parmi les 20 premiers mondiaux et ne représentaient alors que $8,6 \%$ de la capacité mondiale. Le développement de leurs flottes a notamment pu s’appuyer sur les chantiers de construction navale du Japon, de la Corée du Sud et aujourd’hui de la Chine qui à eux trois détiennent en 2003 près de 90\% du carnet de commandes mondial de navires. Seuls les armements européens ont su résister à cette déferlante asiatique grâce à la restructuration drastique d'armements anciens (P\&ONedlloyd ou Hapag-Lloyd) ou grâce à l'émergence d'armements qui innovent tant pour la gestion de leur flotte que dans la constitution de leurs réseaux maritimes et terrestres et qui parviennent même aujourd’hui à contester la suprématie asiatique (Maersk Sea-Land, MSC et CMA-CGM). A l'inverse, les Etats-Unis ne disposent plus de grandes compagnies. Leur rentabilité était jugée insuffisante par les 
groupes auxquels elles appartenaient et elles ont été vendues au cours des années 1990 : Sea-Land au Danois Maersk et APL au Singapourien NOL.

Tableau 1. Les 26 premiers armements mondiaux de transport conteneurisé en 2002, capacité de transport en EVP et nombre de navires

\begin{tabular}{|c|c|c|c|c|c|c|}
\hline Rang & Armement & Pays & EVP & $\%$ & $\begin{array}{c}\text { Nombre } \\
\text { de } \\
\text { navires }\end{array}$ & $\%$ \\
\hline 1 & Maersk Sealand & Danemark & 694940 & 9,2 & 264 & 3,7 \\
\hline 2 & MSC & Italie/Suisse & 391437 & 5,2 & 177 & 2,4 \\
\hline 3 & PONL & RU/PB & 384893 & 5,1 & 147 & 2,0 \\
\hline 5 & Evergreen & Taiwan & 313799 & 4,1 & 122 & 1,7 \\
\hline 4 & APL & Singapour & 260626 & 3,4 & 82 & 1,1 \\
\hline 6 & Coscon & Chine & 247197 & 3,2 & 131 & 1,8 \\
\hline 7 & Hanjin & Corée & 201558 & 2,6 & 53 & 0,7 \\
\hline 8 & K Line & Japon & 169442 & 2,2 & 59 & 0,8 \\
\hline 9 & NYK & Japon & 166964 & 2,2 & 67 & 0,9 \\
\hline 10 & CMA CGM & France & 166872 & 2,2 & 60 & 0,8 \\
\hline & Sous-total & & 2997728 & 40 & 1162 & 16 \\
\hline 11 & MOL & Japon & 157772 & 2,0 & 56 & 0,7 \\
\hline 12 & OOCL & Hong Kong & 155944 & 2,0 & 50 & 0,7 \\
\hline 13 & CSCL & Chine & 149930 & 1,9 & 90 & 1,2 \\
\hline 14 & Hapag-Lloyd & Allemagne & 134009 & 1,7 & 34 & 0,4 \\
\hline 15 & Zim & Israël & 124037 & 1,6 & 56 & 0,7 \\
\hline 16 & HMM & Corée & 123093 & 1,6 & 32 & 0,4 \\
\hline 17 & Yang Ming & Taiwan & 118675 & 1,5 & 40 & 0,5 \\
\hline 18 & Senator & Corée & 107888 & 1,4 & 33 & 0,4 \\
\hline 19 & Lloyd Triestino & Taiwan & 84747 & 1,1 & 24 & 0,3 \\
\hline \multirow[t]{2}{*}{20} & PIL & Singapour & 75692 & 1,0 & 57 & 0,8 \\
\hline & Sous-total & & 4229515 & 56 & 1634 & 23 \\
\hline 21 & Wan Hai & Taiwan & 73885 & 0,9 & 57 & 0,8 \\
\hline 22 & UASC & Koweït & 67922 & 0,9 & 39 & 0,5 \\
\hline 23 & Delmas & France & 52052 & 0,6 & 42 & 0,5 \\
\hline 24 & Safmarine & Danemark & 52028 & 0,6 & 26 & 0,3 \\
\hline 25 & MISC & Malaisie & 49805 & 0,6 & 32 & 0,4 \\
\hline 26 & CSAV & Mexique & 49122 & 0,6 & 25 & 0,3 \\
\hline & Total & & 4574329 & 61 & 1855 & 26 \\
\hline & Flotte mondiale & & 7525058 & 100 & 7118 & 100 \\
\hline & Dt compagnies asiatiques & & 2457017 & 53,7 & & \\
\hline & Dt compagnies européennes & & 1876231 & 41,0 & & \\
\hline & Autres & & 241081 & 5,3 & & \\
\hline & Total & & 4574329 & 100 & & \\
\hline
\end{tabular}

Les ports de l’Asie orientale occupent une place prépondérante dans la hiérarchie mondiale. Sur les dix premiers ports mondiaux classés par la CHTC, neuf sont localisés en Asie orientale. Cinq ports asiatiques arrivent également en tête du classement par le nombre de TEU manutentionnés, les 
ports de Hong Kong et Singapour manutentionnent trois à quatre fois plus de conteneurs que les plus importants ports européens ou américains ${ }^{4}$.

L'Asie orientale concentre $43 \%$ de la capacité mondiale offerte dans ses ports, ce qui la place loin devant les deux autres pôles économiques, l'Europe et l'Amérique du Nord qui totalisent ensemble 38\% de la capacité mondiale offerte. Là encore, l'affirmation de l'Asie orientale comme pôle dominant s’est réalisée au cours des années 1980. Alors que les ports de la Triade concentrent depuis les années 1970 environ 80\% des conteneurs manutentionnés dans le monde, la part de l'Asie orientale est passée de 20\% en 1980 à 46\% en 2000 alors que celles de l'Europe et de l'Amérique du Nord régressaient respectivement de 29 à $23 \%$ et de 23 à $14 \%$.

Figure 3 : Capacité hebdomadaire de transport conteneurisé par port en 2002 (CHTC>50 000 EVP)

\footnotetext{
${ }^{4}$ Il faut cependant nuancer ces écarts. Le transbordement des conteneurs d'un navire à l'autre amène à manutentionner le même conteneur deux fois, et donc à le compter deux fois dans les statistiques portuaires. Or le transbordement représente environ $40 \%$ de l'activité des terminaux portuaires de Hong Kong et 80\% pour ceux de Singapour.
} 


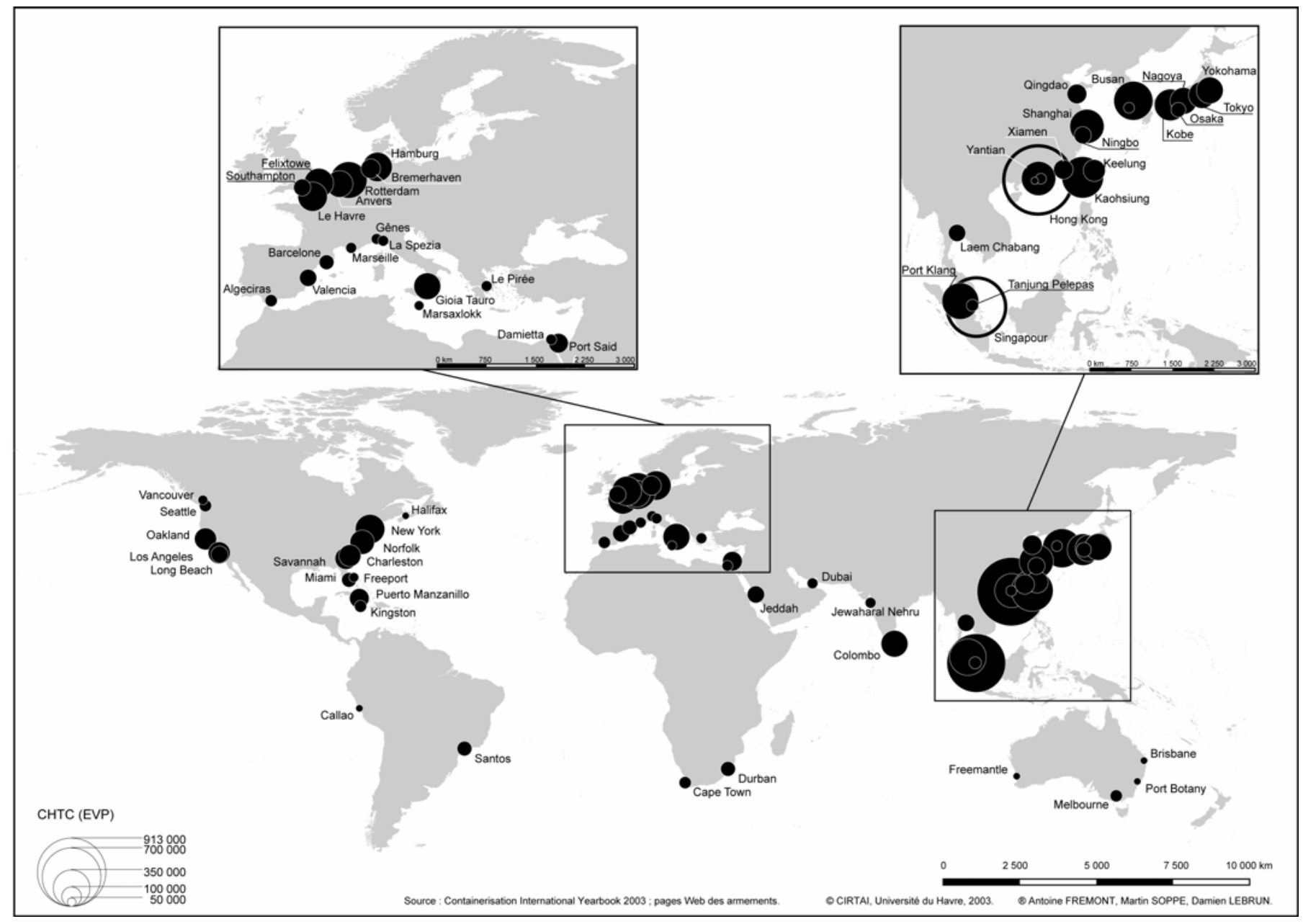




\subsection{La prépondérance des relations Est-Ouest}

La figure 4 schématise la circulation des navires porte-conteneurs à l'échelle mondiale en indiquant le poids relatif de chaque région et de chaque relation dans la capacité de transport offerte par les 26 premiers armements mondiaux. La répartition des capacités entre les différentes relations maritimes place l'Asie orientale au sommet du système mondial de transport maritime conteneurisé : les relations Asie orientale - Amérique du Nord et Asie orientale - Europe sont les plus importantes en termes de CHTC. La nouvelle division internationale de la production confère aux NPI et aujourd'hui à la Chine un rôle d'atelier qui approvisionne en biens manufacturés les pôles économiques traditionnels.

Figure 4. Schéma général de la circulation maritime de trafic conteneurisé
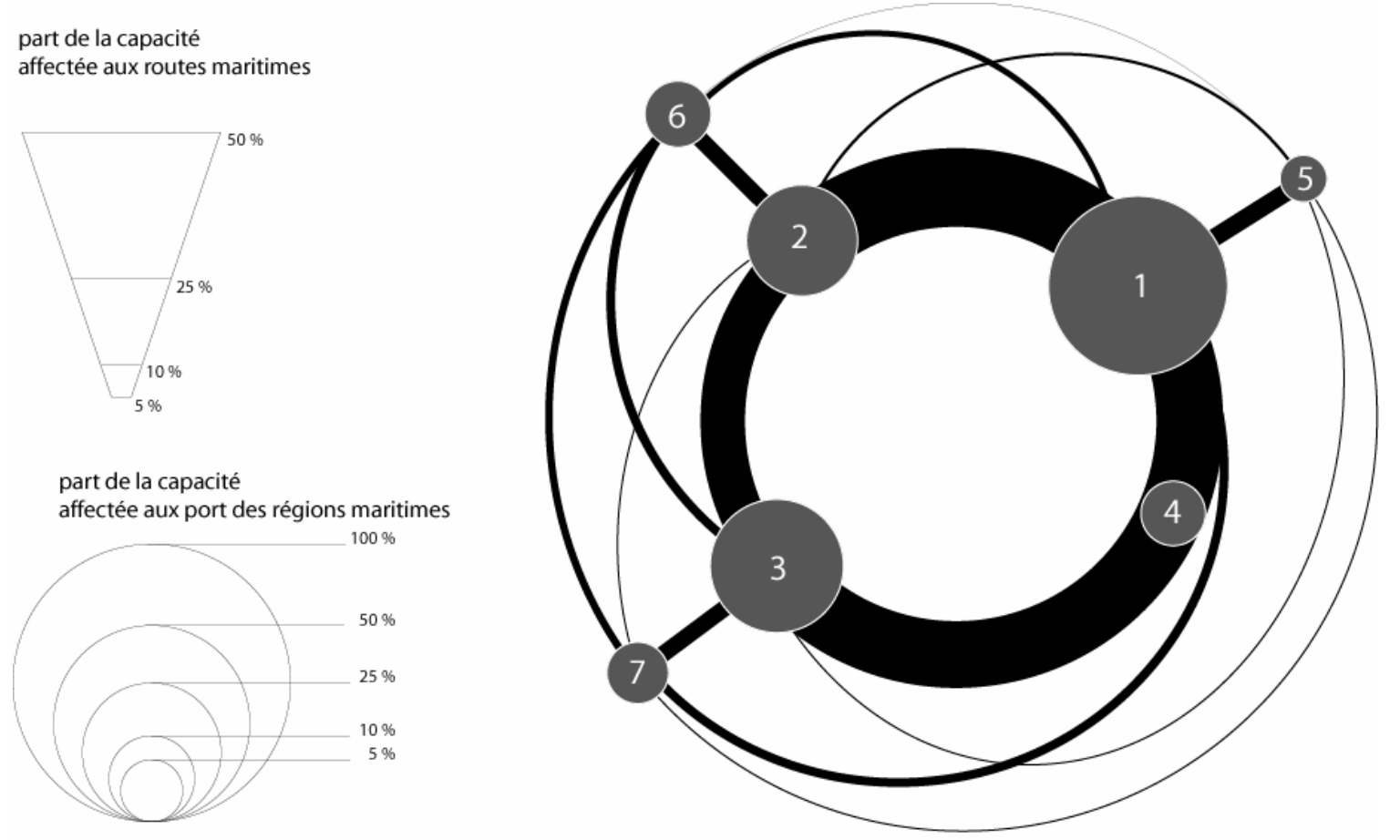

1 - Asie orientale, 2 - Amérique du Nord, 3 - Europe, 4 - Asie du Sud, 5 - Pacifique, 6 - Amérique du Sud, 7 - Afrique

Bien que la CHTC offerte en Europe soit supérieure à celle offerte en Amérique du Nord, ce qui peut s'expliquer par une desserte plus dense des ports européens, on voit apparaître clairement une prédominance des relations transpacifiques entre l'Asie orientale et l'Amérique du Nord alors que le trafic transatlantique entre l'Europe et l'Amérique du Nord devient secondaire: les relations maritimes bipolaires s'affaiblissent relativement 
dans l'Atlantique nord au profit d'un système multipolaire qui voit le trafic transpacifique s'intensifier.

Les relations entre les pôles économiques de l'hémisphère nord sont prédominantes et concentrent $67 \%$ des capacités de transport. Les relations nord-sud entre les pôles de la Triade et les périphéries du Sud atteignent $22 \%$ de ces mêmes capacités. Quant aux relations intra-régionales, elles représentent $8 \%$ de l'ensemble des relations. Les relations entre les périphéries du Sud sont très faibles.

\subsection{La hiérarchie du système portuaire}

La répartition des capacités offertes dans les différents ports desservis par les vingt-six premiers armements est très inégale que ce soit au niveau mondial ou à l'échelle des différentes régions. A l'échelle mondiale, les vingt-sept premiers ports en fonction de la CHTC - soit 6\% du nombre total des ports desservis - concentrent $50 \%$ de la capacité totale offerte. Les plus grands ports sont prioritairement en relation entre eux : 53\% de la CHTC portuaire est constituée de relations mutuelles entre les 50 premiers ports mondiaux, soit environ $10 \%$ des ports étudiés. Les grands ports des trois pôles mondiaux sont prioritairement en relation entre eux. La hiérarchisation au sein de chaque région est également forte. Quelques grands ports concentrent l'essentiel du trafic de chaque rangée. Les plus grandes concentrations mesurées en valeurs relatives ou absolues sont observées pour les trois pôles de la Triade, l'Asie Orientale présentant les taux de concentration les plus élevées.

A l'échelle de chaque rangée maritime, les ports occupent aussi une position inégale bien révélée par la différence de classement entre les ports classés suivant le trafic annuel d'une part et suivant la CHTC offerte de l'autre malgré une corrélation significative entre les deux variables.

La différence entre les deux classements ne remet pas en cause l'utilisation des capacités de transport en tant que moyen d'analyse de la circulation du trafic conteneurisé mais, au contraire, leur explication apporte un élément supplémentaire de compréhension de la hiérarchie portuaire. L'indice d'utilisation des capacités offertes est un ratio entre la CHTC multipliée par 52 semaines et le trafic annuel du port considéré. Il indique si les capacités théoriques de transport offertes font ou non l'objet d'une forte 
mobilisation. Autrement dit, cet indice permet de mesurer si lors de l'escale, un fort volume de conteneurs a été manutentionné proportionnellement à la capacité théorique offerte. Plus l'indice est proche de zéro, plus l'utilisation des capacités offertes par les armements est forte, c'est-à-dire que les navires qui escalent dans ce port sont pratiquement entièrement déchargés puis rechargés. Inversement, plus l'indice s’écarte de zéro, moins l’utilisation des capacités est forte.

Tableau 2: Indice d'utilisation de la CHTC offerte dans les 32 premiers ports mondiaux :

\begin{tabular}{|c|c|c|c|}
\hline Port & $\begin{array}{l}\text { Rang } \\
\text { trafic }\end{array}$ & $\begin{array}{l}\text { Rang } \\
\text { CHTC }\end{array}$ & $\begin{array}{l}\text { Indice } \\
\text { d'utilisation* }\end{array}$ \\
\hline Dubai & 12 & 30 & 0,68 \\
\hline Manille & 22 & 32 & 0,72 \\
\hline Tanjung Priok & 23 & 31 & 0,83 \\
\hline Long Beach & 9 & 26 & 1,01 \\
\hline Los Angeles & 7 & 21 & 1,03 \\
\hline Shanghai & 5 & 8 & 1,45 \\
\hline Laem Chabang & 20 & 28 & 1,50 \\
\hline Busan & 3 & 4 & 1,51 \\
\hline Singapour & 2 & 2 & 1,53 \\
\hline Hambourg & 8 & 14 & 1,57 \\
\hline Anvers & 10 & 17 & 1,57 \\
\hline Bremerhaven & 14 & 24 & 1,58 \\
\hline Tanjung Pelepas & 25 & 29 & 1,68 \\
\hline Qingdao & 17 & 25 & 1,72 \\
\hline Algeciras & 24 & 27 & 1,74 \\
\hline New York & 13 & 19 & 1,76 \\
\hline Rotterdam & 6 & 5 & 1,77 \\
\hline Hong Kong & 1 & 1 & 1,86 \\
\hline Kaohsiung & 4 & 3 & 1,95 \\
\hline Port Klang & 11 & 6 & 2,58 \\
\hline Felixstowe & 15 & 13 & 2,67 \\
\hline Gioia Tauro & 19 & 16 & 2,71 \\
\hline Keelung & 28 & 22 & 2,92 \\
\hline Tokyo & 18 & 12 & 2,98 \\
\hline Colombo & 29 & 20 & 3,34 \\
\hline Yantian & 16 & 7 & 3,42 \\
\hline Yokohama & 21 & 10 & 3,47 \\
\hline Nagoya & 27 & 15 & 3,63 \\
\hline Norfolk & 32 & 23 & 3,77 \\
\hline Oakland & 30 & 18 & 3,79 \\
\hline Kobe & 26 & 9 & 4,03 \\
\hline Le Havre & 31 & 11 & 5,01 \\
\hline
\end{tabular}

* Indice d'utilisation des capacités offertes = $($ CHTC*52 semaines/Trafic annuel en EVP $)$ Source : Containerisation International Yearbook 2003 et base CHTC Le Havre/CIRTAI 
Les ports qui ont un indice inférieur ou égal à 1 ont une utilisation maximale de leur capacité offerte. Ces ports n’ont pas de concurrents directs et permettent la desserte d'une très grosse région métropolitaine. Los Angeles et Long Beach en sont les deux meilleurs exemples. Dubai, aux Emirats Arabes Unis, offre l'exemple d'un port situé dans un environnement particulièrement attractif qui bénéficie d'une expansion exceptionnelle. Manille ou Tanjung Priok, port de Djakarta, sont caractéristiques des ports de feedering dans des pays en voie de développement. Les grands navires mères transocéaniques n’y escalent pas directement afin de ne pas se dérouter de l'axe maritime est-asiatique Singapour, Hong Kong, Busan et pour ne pas pâtir non plus de conditions portuaires qui ne sont pas optimums. Des navires feeders ou nourriciers en provenance de Singapour, de Hong Kong ou de Kaohsiung, ports où s’effectuent les opérations de transbordement entre les navires mères et les navires feeders, les raccordent à l'artère circumterrestre de circulation. Dans ces ports de Manille (2,5M d'EVP en 2003) ou de Tanjung Priok (2,2 M d'EVP en 2001), les trafics sont très intenses : les navires feeders, effectuant les navettes avec le hub de transbordement, y sont entièrement déchargés puis rechargés. Laem Chabang (3,2 M d’EVP en 2003), port de Bangkok, mais avec un degré plus faible d'utilisation des capacités s'inscrit aussi dans cette logique qui révèle la division internationale du travail à l'intérieur même de la zone Asie orientale. Pour ce premier groupe de ports, la très forte utilisation des capacités indique une situation possible de saturation.

Les ports situés généralement au centre des rangées portuaires et à proximité d'un hinterland riche se caractérisent par une bonne utilisation des capacités, comprise entre 1 et 2 . Il s'agit de ports qui dominent le système portuaire et génèrent du trafic : Hong-Kong, Singapour, Busan, Kaohsiung, Shanghai en Asie orientale, Rotterdam, Hambourg et Anvers en Europe et New York en Amérique du Nord.

Cependant, leur utilisation des capacités est moins bonne que la première catégorie car ils doivent compter avec la concurrence de ports qui appartiennent à la même rangée maritime mais sont plus périphériques par rapport au cœur de l'hinterland. Ces derniers présentent les indices d'utilisation les plus faibles. La capacité de transport offerte dans ces ports 
est sous-utilisée : les armateurs y font escale pour compléter leurs cargaisons, mais le nombre d'opérations de chargement/déchargement y reste limité. Il s'agit généralement de ports situés en entrée ou sortie de façade, à proximité d'une grande route maritime. Les ports japonais de Kobe, Nagoya, Yokohama ou Tokyo, ainsi que le port malais de Port Klang sont ainsi des ports d'entrée ou de sortie de l'Asie orientale. Les ports du Havre et de Southampton jouent le même rôle en Europe et les ports de Halifax, Savannah et Charleston en Amérique du Nord. En Asie orientale, Yantian, localisé dans la zone économique spéciale chinoise de Shenzen, concurrence et complète aussi le port de Hong Kong. Appartiennent aussi à cette catégorie des ports de transbordement récents qui sont situés sur l'artère circumterrestre et où les armements effectuent des opérations d'éclatement de leur trafic conteneur. Le port malais de Port Klang tente ainsi de concurrencer Singapour alors que le hub méditerranéen de Gioia Tauro n’a pas la même maturité que celui d'Algeciras, plus ancien. Colombo au Sri Lanka permet une desserte de la péninsule indienne par feeders reliés aux navires mères engagés sur la route Asie orientale/Europe.

\section{L'organisation des routes maritimes par les armements}

\subsection{Une présence globale}

Les 26 premiers armements mondiaux offrent 601 services de transport conteneurisé qui desservent 440 ports répartis sur sept régions maritimes. Dix parmi les armements étudiés sont présents dans les sept régions du monde. Les lignes régulières des vingt-six premiers armements relient toutes ces régions directement entre elles par des capacités de transport.

La couverture globale est organisée de manière rationnelle par les armements grâce à un système de lignes régulières interconnectées où les relations qui desservent deux ou trois continents sont dominantes. Les lignes de cabotage desservant une seule région viennent assurer l'éclatement des flux sur les façades maritimes. Elles sont en nombre élevé et ont une capacité limitée. Il s'agit cependant de marchés spécifiques où opèrent également d'autres armateurs de taille plus modeste que nous n'avons pas pris en considération dans notre étude. 
Les armements offrent les capacités de transport les plus élevées sur les relations Est-Ouest. La desserte des trois pôles principaux est constituée par des services pendulaires reliant deux ou trois continents. Les navires faisant un tour du monde continu sont une exception : trois lignes seulement parmi les six cents lignes étudiées. Les capacités offertes sur l'artère restent relativement bien réparties entre les armateurs : aucun d'entre eux ne détient sur ces relations plus de 20\% des parts de marché. Inversement, les parts de marché sur les relations Nord-Sud peuvent aller jusqu'à plus de $50 \%$ tant le nombre d'armements engagés sur ces routes maritimes secondaires est plus faible, traduisant ainsi une possible tendance vers une situation de type monopolistique.

Cependant, ce constat doit être nuancé. L’artère circumterrestre est dominée par le poids des alliances maritimes qui sont des coopérations techniques entre les armements. Ceux-ci mettent en commun leurs navires sur des liaisons bien identifiées, ce qui leur permet individuellement d'accroître le nombre de ports desservis et les fréquences des services maritimes offerts aux clients. Par contre, chacun conserve son identité commerciale. Grâce aux alliances, les armateurs cherchent à se préserver d'éventuels prédateurs hostiles en atteignant une masse critique suffisante qui leur permet de proposer une desserte globale, d'investir dans des navires de taille plus importante et de partager les risques liés à de tels investissements (Culinane K., Khanna M. 2000). Sur les relations Amérique du Nord - Europe, Amérique du Nord - Asie orientale et Europe - Asie orientale, les parts de marché des quatre plus grandes alliances se montent respectivement à 39\%, 52\% et 61\%. Cependant, l'histoire encore récente de la conteneurisation enseigne que ces alliances ne sont pas nécessairement pérennes.

\subsection{Hiérarchie des routes maritimes et taille des navires}

La hiérarchie est également confirmée par la taille des navires (tableau 7). Les relations Est-Ouest s'opposent très nettement aux relations nord-sud avec des navires aux capacités plus importantes et des fréquences de services où le départ hebdomadaire s’impose comme la norme pour garantir aux chargeurs une grande régularité. Les relations intra-régionales ont, là encore, une place particulière et se placent en dernière position. 


\section{Tableau 3 : La taille des navires et la fréquence selon les relations}

\begin{tabular}{|l|c|c|}
\hline $\begin{array}{c}\text { Type de } \\
\text { relation }\end{array}$ & $\begin{array}{c}\text { Taille de } \\
\text { navire } \\
\text { moyenne } \\
\text { (EVP) }\end{array}$ & $\begin{array}{c}\text { Fréquence } \\
\text { hebdomadaire } \\
\text { moyenne (par } \\
\text { service) }\end{array}$ \\
\hline Est-Ouest & 3686 & 0,8 \\
Sud - Sud & 2199 & 0,7 \\
Nord - Sud & 1804 & 0,8 \\
Intra région & 1029 & 1,1 \\
\hline \multicolumn{2}{|c|}{ Source : base CHTC Le Havre/CIRTAI }
\end{tabular}

Plusieurs facteurs peuvent être invoqués pour expliquer ce phénomène : le degré de compétition sur le marché, l'intensité des échanges entre les différentes régions, le degré de massification et enfin la distance. Les relations est-ouest se caractérisent par une compétition forte et des volumes élevés. L'accroissement de la taille des navires et la concentration des flux permettent de réaliser des économies d'échelle indispensables pour préserver la compétitivité des armements. Les plus gros navires sont déployés sur les services pendulaires desservant les trois pôles économiques.

Les échanges nord-sud sont moins intenses et les flux moins concentrés. De plus petits navires se prêtent davantage à distribuer les flux à partir de l'artère circumterrestre et à accoster dans les ports moins équipés où les temps d'immobilisation sont généralement plus longs. Le marché intra-régional se différencie fortement du marché transocéanique par ses navires de plus petite taille et par un taux de rotation plus élevé. Ces caractéristiques permettent de répondre à une demande de transport géographiquement plus dispersée et sur des distances courtes.

\section{Conclusion}

Le transport maritime conteneurisé participe pleinement au processus de mondialisation car il est le vecteur principal des flux du commerce international, ce qui explique l'étroitesse de ses relations avec l'économie mondiale. Notre étude sur l’offre de transport conteneurisé par les plus grands armements mondiaux, en ne se limitant pas à la seule 
analyse des trafics portuaires, a permis de mettre en évidence l'organisation d'un système de transport lui même global, dominé par les relations EstOuest entre les pôles de la Triade, elles-mêmes polarisées par l'Asie orientale, par les NPIA et aujourd'hui de plus en plus par la Chine qui sont devenus le cœur manufacturier du système économique mondial.

L'organisation actuelle du système mondial de transport maritime conteneurisé s'est mise progressivement en place à partir des années 1980. S'il répond aux besoins du commerce international, il suit aussi les logiques propres à la conteneurisation, notamment en raison des stratégies déployées par les armements. Les ports sont inégalement utilisés en fonction de leur localisation tant par rapport à leur hinterland qu'aux routes maritimes. La concurrence entre les armements s’organise différemment en fonction de l'importance des routes. Le transport maritime conteneurisé, épine dorsale de la mondialisation, constitue un très bon révélateur de l'organisation du monde et de ses inégalités. 